\title{
Globe
}

Revue internationale d'études québécoises

\section{Joe King, Fabled City : the Jews of Montreal, Montréal, Price-Patterson, 2009}

\section{Steven Lapidus}

Volume 13, numéro 1, 2010

Culture et relations internationales

URI : https://id.erudit.org/iderudit/044647ar

DOI : https://doi.org/10.7202/044647ar

Aller au sommaire du numéro

\section{Éditeur(s)}

Globe, Revue internationale d'études québécoises

\section{ISSN}

1481-5869 (imprimé)

1923-8231 (numérique)

Découvrir la revue

Citer ce compte rendu

Lapidus, S. (2010). Compte rendu de [Joe King, Fabled City : the Jews of Montreal, Montréal, Price-Patterson, 2009]. Globe, 13(1), 176-179.

https://doi.org/10.7202/044647ar d'utilisation que vous pouvez consulter en ligne.

https://apropos.erudit.org/fr/usagers/politique-dutilisation/ 
L'après-guerre, avec des initiatives d'éditeurs comme la venue de Sartre ou de Lévi-Strauss à Montréal, connaît des lendemains qui chantent moins. La ressaisie du clergé canadien-français, l'inaction du gouvernement de Maurice Duplessis, réélu en 1944 après une éclipse de cinq ans, et la nonréception du livre canadien en France, voire l'agressivité d'un certain milieu, auquel Robert Charbonneau répond dans La France et nous (1947), secouent le monde de l'édition qui revient presque à la case de départ.

À ce travail de mise en place des connaissances sur le sujet s'ajoute une exceptionnelle créativité en matière de graphisme. La présentation du catalogue, la géniale utilisation pour la couverture d'une affiche de guerre où les baïonnettes sont remplacées par des livres, la mise en caractères plus gros et en rouge des premiers mots des paragraphes comme dans l'enluminure médiévale, les notes de bas de pages regroupées de façon originale tout comme les références bibliographiques compactées d'une manière fonctionnelle poussent le lecteur à aller voir qui est responsable de ce coup de maître. Il s'agit de orangetango, une agence multimédia maintes fois primée de Montréal.

La qualité du travail scientifique et l'inventivité graphique contribuent à la mise en place d'une nouvelle norme d'excellence, d'une nouvelle référence en matière de publication. À nouveau, BAnQ repousse les limites et fait comprendre, après «Le moulin à images» de Robert Lepage, que l'histoire contemporaine, celle du XXI ${ }^{e}$ siècle, doit se faire avec le visuel.

Yvan Lamonde Université McGill

\section{Joe King \\ Fabled City: the Jews of Montreal, Montréal, Price-Patterson, 2009.}

Dans sa plus récente publication, Joe King, journaliste et animateur montréalais de longue date, nous offre un ouvrage divertissant et informatif sur la communauté juive de Montréal. Le livre Fabled City: the Jews of Montreal est le dernier d'une trilogie sur l'histoire de la communauté. Il fut précédé de From the Ghetto to the Main (2000) et de From Baron Byng to Bagels (2006). Se faisant, King confirme sa renommée de conteur et d'homme d'information au sein de la communauté juive montréalaise. 
Tissant anecdotes et événements historiques, King offre une œuvre fascinante et facile à lire sur l'histoire de la première minorité ethnique de Montréal. Ne se satisfaisant pas d'histoires déjà connues où de travaux déjà exploités, King se concentre sur des collections d'archives, des recueils photographiques et des documents pour remettre en lumière des évènements qui forgèrent la communauté juive actuelle. En somme, l'auteur nous propose un recueil léger et bien illustré.

L'ouvrage débute par l'histoire des premiers pionniers juifs, qui arrivèrent en 1759, et s'étend jusqu'à nos jours. Par de courts récits, l'écrivain raconte le développement de la communauté. En en traçant les hauts et les bas, les faillites comme les succès, l'auteur nous en apprend sur l'évolution de la communauté juive ainsi que sur sa contribution au développement de Montréal. L'organisation du livre, à la fois chronologique et thématique, nous permet de choisir la lecture voulue.

La force de l'auteur réside dans sa capacité à rassembler et à organiser des informations, documents et photographies pour appuyer ses histoires. Son travail est truffé de récits d'événements anodins en apparence, mais qui éclairent les dessous de l'apport de cette communauté à l'histoire de la ville. King nous raconte notamment que, dans les années 1960, des représentants du prince Charles se rendirent, à sa demande, à la boulangerie St-Viateur pour se procurer vingt douzaines de bagels.

Il décrit également des personnages importants de la communauté. Par exemple, en expliquant le succès financier sans précédent de la famille Bronfman (tout en rappelant au lecteur que le mot bronfen en yiddish renvoie à une boisson alcoolisée), il souligne le soutien de la famille à des causes juives, au Canada mais aussi à travers le monde. Il raconte en outre que si les Bronfman n'ont jamais illégalement vendu d'alcool aux États-Unis pendant la Prohibition, ils en ont néanmoins légalement procuré à des personnages véreux, tel Al Capone. On apprend par ailleurs l'histoire de la famille Batshaw. Un de leurs fils, Harry, a été juge à la cour supérieure alors que son frère, Manuel, a été directeur de la Fédération CJA (organisation juive caritative montréalaise) et un travailleur social connu pour les efforts qu'il a consacrés à l'amélioration des services sociaux dispensés aux jeunes québécois. De plus, King nous présente d'importants médecins, professeurs, écrivains, hommes d'affaires et philanthropes ayant tous collaborer au développement de l'ensemble des communautés juive et chrétienne. On peut y lire l'histoire de la fondation de l'Hôpital général juif, du sanatorium de Ste-Agathe, ainsi que de plusieurs autres cliniques médicales desservant la population en général. 
S'il met l'accent sur les grands accomplissements de la communauté, King ne fait pourtant pas l'impasse sur des personnages controversés tels le communiste Fred Rose ou le gangster Harry Davis, connu comme le tsar des jeux.

La dernière partie du livre s'avère sans doute la plus forte puisque King y couvre l'époque pendant laquelle il fut lui-même actif comme journaliste. Fort de son expertise, il dépeint les efforts de la communauté pour se remettre des années de guerre, pour faciliter l'intégration des immigrants survivants de l'Holocauste et pour créer les organisations nécessaires au développement des activités communautaires. La croissance et l'épanouissement de la communauté durant ces décennies témoignent, pour l'auteur, d'un rapport très fort à l'identité et d'un fort patriotisme.

Néanmoins, certaines conclusions posent problème, découlant parfois d'opinions politiques que King laissent transparaittre, comme dans le cas suivant:

Quebec nationalists claim that Louis-Joseph Papineau called for and led the rebellion [of 1837]. In fact, essential to the separatist cause is the fiction of Les Patriotes - French Canadians rising up against their English masters. It is simply not true. Papineau certainly called for more freedom for Canadians - but when violence started, called for by Dr. Nelson and his brother Robert, with Anglophones very much in the forefront, Papineau fled to the United States, and France, and remained there until an amnesty allowed him to return, without penalty after ten years. (p. 31)

Si l'implication de Papineau, au début de la révolution armée, fut minime et sa fuite rapide, il n'en dédia pas moins les années qui suivirent à la coordination du mouvement Patriote, qui, contrairement à ce que prétend King, participa à une réelle lutte armée contre les autorités coloniales anglaises. King ignore également les efforts déployés en France par Papineau pour améliorer la situation de ses compatriotes du Bas-Canada. Ajoutons que si la grande majorité des Juifs de l'époque s'identifièrent à la couronne anglaise, certains appuyèrent tout de même la rébellion. Ezekiel Hart, entre autres, organisait des réunions de Patriotes à son domicile.

On peut aussi critiquer King pour son filio-piétisme - son acceptation sans réserves des histoires de ses ancêtres. Effectivement, certaines annales sont plutôt mal documentées alors que d'autres reflètent un manque d'esprit critique envers les contributions de la communauté. Comme l'indique le titre de son ouvrage - ainsi que celui des autres livres qui complètent la trilogie -, le principe unifiant est l'importance de la commu- 
nauté juive de Montréal. À priori, une telle position, qui peut influencer l'interprétation des sources historiques, est généralement évitée par les historiens. Un autre indicateur de la nature parfois démagogique de ce texte est l'usage excessif du point d'exclamation, une pratique rare dans les ouvrages plus formels.

Quoique Fabled City soit un recueil fascinant d'anecdotes, de faits et d'opinions, sa valeur historique n'est pas constante. Si King offre une bibliographie, l'absence de références dans le corps du texte rend néanmoins difficile toute vérification de renseignements. En revanche, la présence de nombreuses photographies et documents donne vie aux récits, et contribue à faire de Fabled City une importante introduction à la culture populaire des Juifs montréalais.

Steven Lapidus Université Concordia

\section{Jose del Pozo \\ Les Chiliens au Québec. Immigrants et réfugiés, de 1955 à nos jours, Montréal, Boréal, 2009.}

La communauté chilienne occupe une place remarquable dans la société québécoise, non pas par sa taille ou son influence, mais pour des raisons historiques et sociologiques particulières qui, d'ailleurs, permettent d'éclairer l'évolution des rapports entre la majorité et les minorités. Il va de soi que toute étude de cas d'un groupe issu de l'immigration est pertinente et le cas des Chiliens, même s'il est relativement mieux connu que d'autres, méritait bien qu'un ouvrage entier lui soit consacré. Outre les spécialistes, bien des lecteurs québécois tireront profit d'un livre qui retrace avec minutie la trajectoire de cette fascinante communauté, traversée par des enjeux politiques, économiques et culturels. José del Pozo, lui-même d'origine chilienne, a vécu cette trajectoire de l'intérieur et, bien évidemment, en possède une connaissance intime. Ses indubitables qualités d'historien donnent au travail la rigueur nécessaire, sans laquelle ce type de portrait aurait pu sombrer dans la description sèche, sans profondeur analytique. En fait, dans le but de maintenir une distance convenable vis-à-vis de son objet, l'auteur a constitué un ample échantillon de répondants et s'est appuyé sur 